\title{
http://artnodes.uoc.edu
}

\section{MediaLab Madrid 2002-2006 Cultura participativa y activismo social en Madrid}

\author{
Raquel Caerols Mateo \\ Universidad Francisco de Vitoria \\ Beatriz Escribano Belmar \\ Universidad de Castilla-La Mancha \\ Fecha de presentación: enero de 2019 \\ Fecha de aceptación: mayo de 2019 \\ Fecha de publicación: julio de 2019
}

\section{Cita recomendada}

Caerols Mateo, Raquel; Escribano Belmar, Beatriz. 2019. «Medialab Madrid 2002-2006.Cultura participativa y activismo social en Madrid». Artnodes. N. ${ }^{\circ} 24:$ 111-120. UOC [Fecha de consulta: dd/mm/aa]. http://dx.doi.org/10.7238/a.v0i24.3273 \footnotetext{
@ $C$ Los textos publicados en esta revista están sujetos -si no se indica lo contrario- a una licencia de
SOCMERIGHISRESERVED
Reconocimiento 4.0 Internacional de CreativeCommons. La licencia completa se puede consultar en
https://creativecommons.org/licenses/by/4.0/deed.es_ES.
}

\section{Resumen \\ Iniciado el siglo xxı, potenciado por el contexto digital, la participación ciudadana comenzó a ser central para la sociedad contemporánea. En el campo artístico, eso se tradujo en la generación de nuevas formas artísticas de acción social y participación ciudadana. El mejor reflejo de esta realidad será la aparición de centros o medialab, donde al diálogo entre el arte, la ciencia y la tecnología se sumará la dimensión sociedad, como el pionero MediaLab Madrid, inaugurado en 2002 y que se convirtió en paradigma de acción, cultura participativa e integración social. Este artículo formaliza un recorrido histórico por la creación de este centro y proyecto emblemático y analiza su propuesta como un lugar donde dar cabida a formas emergentes de participación y transformación social, en relación a su programa de exposiciones, talleres, seminarios y simposios, conferencias, encuentros y acciones durante el período 2002-2006, liderado por}




\title{
artnodes
}

http://artnodes.uoc.edu

MediaLab Madrid 2002-2006

sus creadores, Karin Ohlenschläger y Luis Rico. Dicha programación muestra una metodología innovadora de propuestas artísticas y espacios de reflexión y su diálogo con la tecnología como herramienta de activismo social y transformación. Este análisis está basado en entrevistas realizadas a los protagonistas que idearon este proyecto, así como a investigadores y artistas de relevancia internacional, que aportan una mirada externa de su impacto.

\section{Palabras clave}

sociedad, activismo, arte, ciencia, tecnología, medialab

\section{Medialab Madrid 2002-2006 \\ Participatory culture and social activism in Madrid}

\begin{abstract}
Citizen participation is a crucial element to contemporary society at the start of the $21^{\text {st }}$ century. In the artistic field this has been translated into generating new artistic forms of social action and citizen participation. This reality is best reflected in the emergence of centers where "society" is added to art, science and technology dialogue, such as the pioneering MediaLab Madrid center inaugurated in 2002 as a paradigm of action, participatory culture and social integration. This article formalizes a historical tour of how this center and emblematic project was created. It then analyzes the center's purpose as a place to accommodate emerging forms of participation and social transformation in relation to its program of exhibitions, workshops, seminars and symposia, conferences, meetings and actions during the 2002-2006 period when it was led by its creators, Karin Ohlenschläger and Luis Rico. The program reveals an innovative methodology of artistic proposals and spaces for artistic reflection and dialogue with technology as a tool for social activism and transformation. This study is based on interviews with the actors who devised this project, as well as internationally renowned researchers and artists, who provide an external view of the center's impact.
\end{abstract}

\section{Keywords}

society, activism, art, science, technology, medialab

\section{Introducción}

A finales del siglo xx, los medialab, basados en el diálogo entre el arte, la ciencia y la tecnología comenzaron a ser fundamentales dentro del contexto histórico-cultural, simbolizan el paso de la acción individual a la colectiva, y se traducen en la acción social y la creación artística, la participación ciudadana y las nuevas formas educativas. Se trata de un colectivismo que es una renovación proveniente de las vanguardias históricas y que pudo percibirse más nítidamente desde finales de los años sesenta, ya que los centros ciudadanos comenzaron a emerger como un claro símbolo de ese fenómeno cultural (Deitcher 2000).

El término medialab se ajusta a un espacio o laboratorio donde se trabaja colectiva e interdisciplinarmente, con actividades y producciones que pueden situarse entre el activismo social, la producción artística, o la alfabetización ciudadana en las nuevas tecnologías (Serra 2010). Por ese motivo, el florecimiento de los medialab también puede relacionarse con la llegada al mercado de diversas tecnologías de la imagen a partir de los años sesenta del siglo pasado. Estas tecnologías, que alcanzaron el mercado con precios desorbitados para el bolsillo de un artista, se ubicaron en diversos medialab, donde fueron accesibles para poder crear con ellas. Es el caso del MIT Media Lab, que se fundó en la Escuela de Arquitectura y Planificación del Massachusetts Institute of Technology (MIT) en el año 1985, aunque estaba más dirigido a las empresas y menos al activismo social (Fagerstrom, Arntzen, y Foxall 2015).

A pesar de que en España se hicieron algunos intentos que no Ilegaron a formalizarse como tales, como Arteleku, MIDECIANT, Hangar o Laboratorio de Luz, MediaLab Madrid abogó por la autoría colectiva, los proyectos con impacto en la sociedad y para la sociedad, y donde el ciudadano tuviera el protagonismo. Este fue un proyecto concebido y dirigido por Karin Ohlenschläger y Luis Rico desde el año de su inauguración en 2002, dentro de una colaboración entre la 


\section{artnodes}

http://artnodes.uoc.edu

MediaLab Madrid 2002-2006

productora cultural Todo Fluye S.L. y el Centro Cultural Conde Duque del Ayuntamiento de Madrid. Las personalidades de Ohlenschläger y Rico fueron tan significativas que se ha llegado a decir que MediaLab Madrid se distinguía por «[...] la intensa presencia carismática de Luis y Karin y la idea de que el Medialab seguía una gran narrativa. Una narrativa compleja y por naturaleza diversa e inclusiva, pero una narrativa, al fin y al cabo» (Ortiz 2018).

Karin Ohlenschläger confiesa que entre sus primeras referencias nacionales para este proyecto estaban los seminarios y colaboraciones entre artistas, músicos, poetas, arquitectos e informáticos que tuvieron lugar en el Centro de Cálculo de la Universidad de Madrid, donde se aplicó la automatización de las formas a áreas, como las artes plásticas, la educación, la música o la literatura (VV. AA 2002); y la experiencia de Espacio $\mathrm{P}$-donde había trabajado durante cinco años- como espacio autogestionado por artistas «artist-run space» (Blessi, Saccoy Pilati 2011, 141-166) en torno a prácticas artísticas interdisciplinares y multimedia, entre principios de los años ochenta y mediados de los noventa.

Este proyecto planteaba ser una «plataforma estable» (Rico 2018) y un catalizador de la cultura digital en la ciudad, que asumía una función social con un amplio programa de actividades en torno a la investigación, la formación, la producción y la mediación, pero basado siempre en un diálogo entre el arte, la ciencia, la tecnología y la sociedad (Sánchez 2011; Del Río 2003). Aquel contexto es descrito por Luis Rico del siguiente modo:

«En 2001 la realidad sociocultural de Madrid constituye un hervidero de creatividad, pero sus instituciones culturales, salvo algunas excepciones, eran insensibles a las necesidades de los artistas, colectivos e investigadores locales relacionados con la cultura digital y las prácticas artísticas emergentes» (Rico 2018).

MediaLab Madrid siempre contó con una participación masiva de la ciudadanía, inclusive, de diversos colectivos, movimientos makers (Anderson 2013) y movimientos sociales, siendo el concepto colectivo un pilar sobre el que se constituyó. Además, y referenciando el Centro de Cálculo, se convirtió en un lugar donde se encontraron personalidades de diversas áreas, que transformaron MediaLab Madrid en un lugar para la investigación, la formación, la mediación y la práctica interdisciplinar.

Por todo lo anterior, este artículo formaliza un recorrido histórico acerca de la creación de este centro sobre el sustrato epistemológico, conceptual e histórico en el que se fundamentó el proyecto, un recorrido por la historia de los medialab como lugares simbólicos de un periodo, para que MediaLab Madrid pueda ser contextualizado como pionero en el ámbito español y como impulsador de diversas prácticas que tuvieron el respaldo de las instituciones públicas (Chatzichristodoulou 2013, 301-318).

\section{Metodología}

La metodología se ha diseñado en relación con dos líneas de trabajo. Por un lado, se construye un relato que fundamenta las bases epistemológicas y conceptuales del concepto medialab, sustentado en la relación entre el arte, la ciencia y la tecnología, así como el recorrido histórico-social que da sentido a su presencia central en las sociedades contemporáneas. Por otro lado, se utiliza el estudio crítico de la información sobre el centro durante el período transcendental entre 2002-2006. Se realiza un breve recorrido por el programa desarrollado en esos años, centrado en aquellos talleres, exposiciones y simposios que tuvieron especial relevancia en las diferentes formas de arte y acción social, y cuya selección ha sido realizada por Ohlenschläger. El citado estudio de caso se fundamenta, además, en una serie de entrevistas detalladas en el cuadro 1, donde se hace distinción entre los protagonistas y aquellos artistas, investigadores $y / 0$ científicos externos a este proyecto.

\section{Entrevistas. Protagonistas de MediaLab Madrid}

Karin Ohlenschläger. 12/02/2018

Luis Rico. 25/02/2018

\section{Preguntas formuladas}

¿Por qué surgió MediaLabMadrid? Necesidad en el contexto

¿Cómo surgió el proyecto? ¿Fue un encargo institucional?

¿Quién inició el proyecto?

¿Quiénes fueron los agentes y/o personas implicadas?

¿Cuáles fueron los centros referentes en Europa?

En el contexto español, ¿este proyecto fue pionero en sumar «Sociedad» a la interrelación entre el arte, la ciencia y la sociedad?

Entrevistas. Personalidades con implicación directa y/o indirecta en MediaLab Madrid

José Ramón Alcalá. 29/01/2018

Pau Alsina. 09/02/2018

Ricardo Iglesias. 31/01/2018

Kepa Landa. 21/02/2018

Santiago Ortiz. 21/02/2018 


\section{artnodes}

\section{Preguntas formuladas}

Sobre el impacto de MediaLab Madrid en las políticas culturales del Ayuntamiento, en el arte y la cultura en general. ¿Qué nuevos modelos, formatos y conceptos introdujo MediaLab Madrid en el arte y la cultura de principios del siglo $x \times 1$ ?

¿Qué significó MediaLab Madrid para la escena del arte de los medios? ¿Qué aportó al diálogo entre el arte, la ciencia, la tecnología y la sociedad?

¿Qué significó MediaLab Madrid para la internacionalización de los artistas de arte de los medios españoles?

Papel del visitante/usuario en este nuevo contexto implementado por MediaLab Madrid. ¿Generó nuevas audiencias?

Cuadro 1. Entrevistas y preguntas formuladas

Utilizando los métodos citados, se ha tratado de obtener conclusiones que permitan delimitar la vinculación de éste, no sólo con el aspecto social, sino con el arte de los medios y el papel en la educación y participación social en torno a las tecnologías digitales.

\section{Fundamentación conceptual e histórico-social del medialab}

Si ha habido una propuesta en el campo de la creación artística en la que la acción social y el diálogo entre el arte, la ciencia y la tecnología se fusionaran bajo el epígrafe las dinámicas fluidas, fue en el Encuentro Europeo de Medialabs «Toma las riendas», dirigido por el artista Daniel García Andújar, que inauguró el proyecto MediaLab Madrid dentro del I Festival Internacional de Arte, Ciencia y Tecnología, Cibervisión en el año 2002. La contundente expresión «Toma las riendas» fue una declaración de intenciones en el proyecto, que la crítica y comisaria Karin Ohlenschläger y el artista y productor cultural Luis Rico pusieron en marcha. Este primer festival incluía una exposición de arte electrónico y un Ciclo de Invierno de Ciencia y Tecnología de la Fundación Complutense, que estaba dirigido por el bioquímico Federico Morán.

Si atendemos al sustrato epistemológico, conceptual e histórico sobre el que se sostiene este proyecto, observaremos que su primer asentamiento está en el diálogo y dialéctica entre el arte, la ciencia y la tecnología, que se inició con el nacimiento del pensamiento moderno.

En la memoria ${ }^{1}$ que Ohlenschläger desarrolló sobre MediaLab Madrid durante los años 2002-2006, afirma que «La interacción entre nuestro imaginario y las tecnologías de la comunicación constituyen uno de los motores de la producción cultural» (Ohlenschläger 2007, 9).
El sentido que muestra esa afirmación marca la constante que tejió el nacimiento del pensamiento moderno en el diálogo establecido por los artistas con las tecnologías de su tiempo y su imaginario, los mundos posibles y simbólicos.

Asimismo, en la citada memoria encontramos reflexiones que consolidan nuestra fundamentación cuando dice: «[...] una evolución que nos ha llevado desde la edad de la piedra a la era del silicio (que básicamente sigue siendo arena muy depurada) y de la información» (Ohlenschläger 2007, 10). Tecnificación de la estética o humanización de la tecnología son así identificadores del pensamiento moderno, ¿en qué dirección va si no la afirmación que encontramos en dicho dossier?: «Ciencia y tecnología también son cultura. Ambos tienen un papel incuestionable en la manera de concebir, percibir y relacionarnos con el mundo que nos rodea; influyen en nuestros hábitos y costumbres» (Ohlenschläger 2007, 10).

Adentrándonos en lo epistemológico, el constructo arte, ciencia y tecnología nos conduce a comprender el arte como una forma de conocimiento al mismo nivel de la ciencia, y en esa tensión epistemológica se centran los debates entre el arte, la ciencia y la tecnología: «La ciencia al igual que el arte, aunque de distinta manera, está intentando hacer visible lo invisible. Está intentando generar distintos modelos de cognición y comprensión, distintas maneras de entender los patrones y procesos que rigen la vida en general y la experiencia contemporánea en particular» (Ohlenschläger 2007, 9-10).

No es casualidad, pues, que esas sean las bases conceptuales que Ohlenschläger cita en su presentación durante el encuentro LabMeeting 2015 Madrid, celebrado del 22 al 24 de septiembre en Medialab Prado, y que también son señaladas por Luis Rico en la entrevista realizada en 2018 con motivo de esta investigación:

- Biosfera, Noosfera., de V. I. Vernadski.

- Teoría general de sistemas, de L. Bertalanffy.

- Cibernética, de N. Wiener y G. Bateson.

- Las estructuras disipativas, de I. Prigogine.

- Autopoiesis, Autogestionarse como individuos, como colectivo, como ciudad, de F. Varela y H. Maturana.

- Teoría de la comunicación, Sistemas autorganizados, de M. McLuhan.

- Simbiogénesis, la evolución de la vida por cooperación entre los sistemas, de L. Margulis.

- Complejidad y transversalidad, de E. Morin.

- Sociedad red, de M. Castell.

Una vez se asentó el diálogo entre el arte, la ciencia y la tecnología como método para la construcción del conocimiento, ¿qué sucedió para que se fracturase el establishment de las academias y los

1. Para ampliar, véase: https://www.medialab-prado.es/sites/default/files/import/ttp_medialab/16/16682/16682_10.pdf. 


\section{artnodes}

http://artnodes.uoc.edu

MediaLab Madrid 2002-2006

salones oficiales, y se iniciara así una crisis arte-sociedad, que se traduciría en el protagonismo de la sociedad en su diálogo con el arte? Diferentes hitos marcan éste como un segundo giro de la modernidad.

El desmantelamiento de las academias será el acicate para la conquista de la autonomía y la libertad creadora que dio lugar a los ismos de las vanguardias. Esa salida de las instituciones llevó directamente al credo que fundamenta los manifiestos de todos esos movimientos de vanguardia: el arte para la sociedad y todas las esferas de la vida:

«Democratizar el sistema del arte, sacándolo a la calle para llegar al gran público, lo que obligaba a que abandonase su hábitat tradicional (el museo y las galerías) y, por tanto, el elitismo que secularmente había caracterizado su consumo» (Salaris 1990, 180-197).

En ese contexto, no es de extrañar que podamos encontrar ya incipientes labs a comienzos de los años veinte del siglo xx, como el Laboratorio Experimental de Construcciones Cinéticas del Proletkult de Moscú ${ }^{2}$ (Ortega y Villar 2014, 153). Este devenir guarda una relación directa con la desestructuración del establishment del arte: los artistas llevan el arte a la sociedad, lo que implica en el mismo devenir de los acontecimientos el giro en los procesos de creación, para pasar en ese nuevo discurso arte-sociedad a la cocreación, al arte participativo, colaborativo (Rodrigo 2007; Rodrigo 2011), que conducirá al binomio arte-vida de Fluxus (Mullinax 2014, 112). En las palabras de Rico se evidencia el relato que llega hasta MediaLab Madrid: «Se concibió como un proceso de investigación e innovación social y cultural que exploraba el binomio arte-vida a la luz de los avances científicos y tecnológicos en relación con los lenguajes y prácticas emergentes, así como con los correspondientes movimientos y dinámicas sociales» (Rico 2018).

Las reflexiones de los investigadores Ortega y Villar en el artículo «El modelo Media Lab: contexto, conceptos y clasificación» (2014) refuerzan y evidencian estos planteamientos, la génesis que subyace en el concepto de medialab y que describe una línea directa con el camino iniciado por los artistas de finales del siglo xIX y los artistas de las vanguardias: «El concepto de colaboración (Laddaga 2011; Rodrigo 2011; Ardenne 2006), tal cual lo entendemos en nuestra investigación, está caracterizado por traspasar las estructuras de producción y distribución destinadas a las élites y abrirse al ámbito público» (Ortega y Villar 2014, 151). Así pues, la génesis del concepto lab está en su diálogo con la sociedad y vinculado al contexto social en el que se imbrica. Se trata de una colaboración y una participación, de un activismo social en un proceso donde el ciudadano se empodera en la potestad de conformar el sentido que entiende que deben atribuir a las tecnologías de la comunicación (Haste 2009, 207-223).

Así, la conjunción arte-vida alcanza su máximo esplendor en la década de los sesenta, con movimientos como Fluxus y figuras como Wolf Vostell; siguen con la generación de conceptos como prosumer (Toffler 1980), cocreación, o procomún, entre otros, como resultado natural en un espacio red en su diálogo con la tecnología.

De este modo, estamos en disposición de precisar la génesis de un medialab, en un contexto de acción social, arte-acción, activismo, cambios en los procesos de creación en las artes, prosumer, arte colaborativo, cocreación, procomún, creación en red, aprendizaje no formal o redes de aprendizaje, entre otros (Estalella, Rocha, Lafuente 2013; Ortega, Rodríguez 2011).Todo ello, como resultado de los cambios que se produjeron en los procesos de creación en el segundo giro de la modernidad, y que alcanzan su expresión exponencial en la consecuente y necesaria creación de los laboratorios de medios. La descripción que realiza Ohlenschläger en su intervención en el encuentro de medialab sobre la ideación y diseño, que conformó la concepción de MediaLab Madrid, permite comprender la expresión final del cambio de paradigma que se produce en las artes desde finales del siglo xIx hasta nuestra contemporaneidad (Brea 2002):

- Generar estructuras abiertas de trabajo en red

- Habilitar espacios polivalentes

- Ofrecer visibilidad a los procesos de investigación y producción

- Construir vasos comunicantes entre distintos campos de conocimiento

- Explorar zonas fronterizas entre sistemas biológicos, sociales, tecnológicos y culturales

- Crear comunidad

- Propiciar equipos interdisciplinares

- Plantear temas transversales

Esa transformación también se evidencia poniendo atención a los antecedentes que señaló Ohlenschläger y que precedieron al proyecto de MediaLab Madrid. En ellos se observan las diversas tipologías de medialab, donde no solo prima el carácter social, sino también la vinculación con la empresa o la universidad (Romero-Frías y Robinson García 2017) con ese hilo conductor en relación con el giro señalado en las prácticas artísticas:

- Bauhaus, Weimar, Dessau, Berlín, 1919-1933

- Black Mountain Colleague, 1933-1957

- Fluxus, 1961

- MIT Center for Advance Visual Studies (CAVS), Boston, 1967

2. En dicho laboratorio, se trató de activar los nuevos medios de comunicación en espacios colectivos experimentales para organizar una producción artística diferente de la elitista.

3. Para ampliar, véase: https://ebuah.uah.es/dspace/bitstream/handle/10017/21146/Modelo\%200rtega.pdf?sequence=1. 


\section{artnodes}

http://artnodes.uoc.edu

MediaLab Madrid 2002-2006

- Seminarios del Centro de Cálculo de la Universidad de Madrid, 1968-1973

- The Kitchen, Nueva York, 1971-73

- Espacio P, Madrid, 1981-1997

- Ars Electrónica, Linz, 1979

- MIT Medialab, 1985

- ZKM, Karlsruhe, 1989

- Planetary Collegium, Plymouth University, 1994

En este listado podemos constatar que existen perfiles más académicos, perfiles profesionales, 0 aquellos más cercanos al concepto de centro cultural o de carácter cívico. Sin embargo, lo que define a todos ellos son sus procesos, la colaboración, la participación y la transversalidad. MediaLab Madrid se diferenció por su dimensión cívica y social, y constituyó no solo un hito nuevo en el panorama de la creación y cultura de Madrid sino que, podríamos decir, a escala nacional. Tal es así que fue el primer centro que introdujo en España el hardware Arduino y Processing en su programa de talleres y exposiciones, a modo de ejemplo.

La organización, en el marco de Cibervisión del Encuentro Europeo MediaLabs «Toma las riendas», aportó una visión panorámica en la que participaron expertos, artistas y activistas europeos, como Inke Arns (Berlín), Heath Bunting (Londres), David Casacuberta (Barcelona), Thomax Kaulmann (Berlín), Eric Kluitenberg (Ámsterdam), Sebastian Luetgert (Berlín), Dirk de Wit (Bélgica) y Simon Worthington (Londres). Es por ello que el caso de MediaLab Madrid se presenta como paradigmático para estudiar nuevas formas de participación ciudadana en el contexto digital, en la acción social, en el arte acción o en el aprendizaje no formal (Sangrà, Wheeler 2013; Rosales 2009). De hecho, un recorrido por los cinco años de programa (2002-2006) evidencia el papel central que tuvo en las expresiones de creación artísticas y las cuestiones señaladas.

\section{Un recorrido por cinco años de la programación del proyecto MediaLab Madrid}

De las múltiples propuestas, nos vamos a centrar en aquellas que destacan por su intencionalidad en el activismo social y la cultura participativa, teniendo en cuenta que este listado ha sido supervisado por Ohlenschläger. Las explicaciones y particularidades de cada taller se pueden encontrar en dicho dossier.

— Exposiciones. La dinámica de sala de exposiciones se rompe para convertirse en espacios que conectan con las calles, los ciudadanos y el entorno cercano. Muchas de las propuestas eran participativas y/o interactivas, en el sentido de coproducción con el ciudadano/ espectador prosumer. Ejemplo de ello son:
- Cibervisión 02_dinámicas fluidas

- Economías alternativas, sociedades alternativas

- Narrativas y procesos de creación artística fundamentadas y sustentadas en la acción social.

— Talleres. Ejemplos significativos son:

- ¡Toma las riendas!

- Individual Citizen Republic Project

- Regreso al futuro de los procomunes. Taller de copyleft

- $3^{\text {er }}$ taller de tecnologías del cuerpo: tecnopoder y lenguajes emergentes

- Arduino: herramientas open hardware

- Taller Bordergames

- ¿Interactivos?

— Seminarios y simposios, entre los cuales destacan:

- Cibervisión02. Toma las riendas / Nuevos espacios en la comunidad artística (Encuentro Europeo de Medialabs)

- banquete03. Redes sociales y redes de comunicación

- Conferencias, presentaciones y encuentros, entre los cuales señalamos:

- Conferencia y debate: Cambio climático y Ecolocación Algorítmica

- Conferencia y debate: Ciencia, arte y activismo

- Prácticas artísticas independientes en Madrid

- Prácticas artísticas en Madrid

— Acciones e intervenciones. Ejemplo de ello son:

- banquete03. Truisms. Jenny Holzer

- banquete03. Cocina solar. Wolfgang Scheffler, Andreas Wegner y Fundación Terra

- banquete03. PANlingua. Antoni Miralda / Food Culture Museum 


\section{artnodes}

\section{Medialab a vista de cifras}

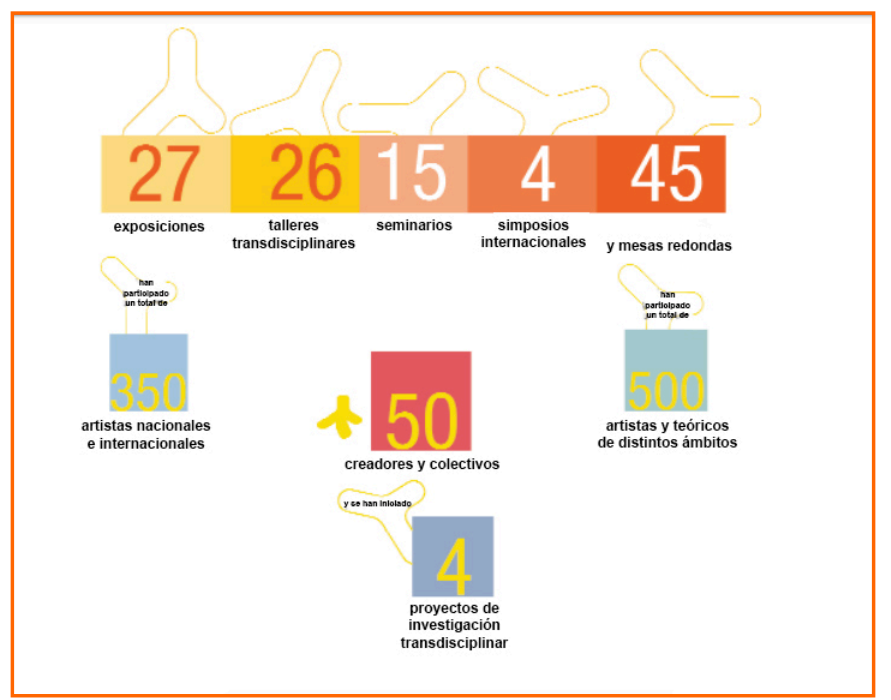

Figura 1. Actividades y participantes

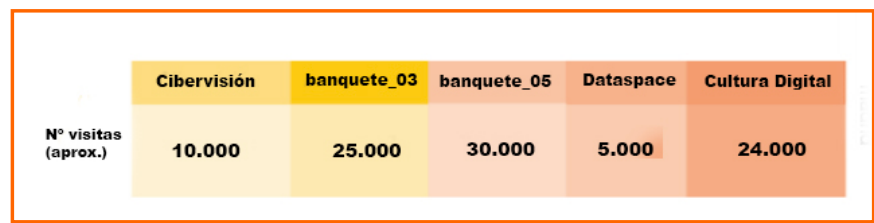

Figura 2. Número de visitas a las exposiciones

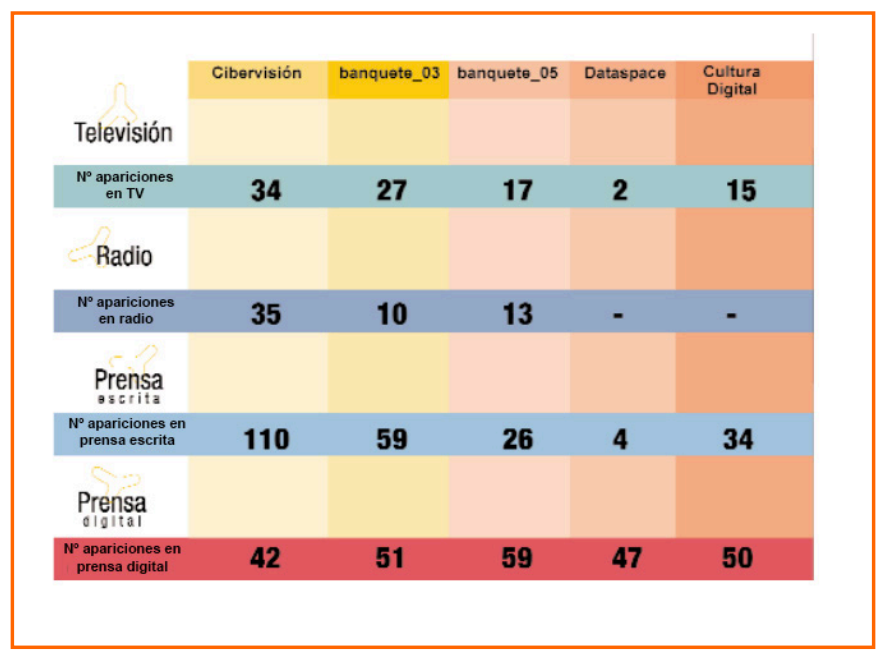

Figura 3. Presencia en medios de MediaLab Madrid

\section{Conclusiones: Repercusión de MediaLab Madrid en el panorama artístico contemporáneo y en las nuevas formas de aprendizaje}

Analizar la repercusión que MediaLab Madrid tuvo en las nuevas formas de aprendizaje propiciado por las nuevas tecnologías parte de la siguiente afirmación de Ohlenschläger:

«Ante los imparables avances de la era digital, nos pareció muy urgente articular un espacio y un programa público estable que no solo se hiciera eco de lo que estaba pasando, sino que participara muy activamente en este proceso de transformación [...]» (Ohlenschläger 2018).

Así, se convirtió en un espacio para la normalización de toda esta transformación y participación social desde el arte y la cultura, con reflexiones y debates, programas formativos y educativos, investigaciones, producciones y exposiciones. Gracias a todo ello, no sólo MediaLab Madrid se convirtió en epicentro del arte y las nuevas tecnologías, sino que a escala nacional era un punto obligado para aquellos interesados en las nuevas prácticas. Según las palabras de Pau Asina, este centro fue:

«[...] un actor especialmente relevante en el panorama internacional, por la capacidad que tuvo de articular proyectos que vertebraron la acción de muchos creadores, investigadores, académicos o profesionales trabajando en la relación entre arte, ciencia, tecnología y sociedad, y más allá» (Alsina 2018).

Supuso un punto y aparte en la relación de interdisciplinariedad, 0 a-disciplinar (Alsina 2018), y se convirtió en un ejemplo de lo que significaba dejar atrás los centros más academicistas, tal y como estaba ocurriendo en otros países como Estados Unidos. MediaLab Madrid desarrolló:

«[...] un programa de múltiples enfoques, donde se entremezclaban retroalimentariamente el taller-lab de experimentación, los seminarios de innovación y los encuentros, exposiciones y festivales interdisciplinares, juntando en un mismo debate a científicos, creadores y artistas audiovisuales» (Alcalá 2018).

El impulso que recibieron diversos artistas a través de su participación en este centro también fue fundamental. Pablo Valbuena o Ricardo Iglesias son algunos ejemplos cuyo paso fue cardinal para desarrollar su carrera artística, pues consiguieron conectar y colaborar con otros creadores internacionales. Pero también sucedió lo contrario, ya que el contexto internacional comenzó a conocer a los protagonistas, los proyectos y demás participantes nacionales.

En la primera etapa de este espacio, participaron: Kepa Landa, como coordinador técnico, Sonia Diez Thale, como coordinadora del 


\section{artnodes}

programa de actividades y Sylvia Molina, como asesora y colaboradora del programa educativo. Posteriormente, se unieron otros, como el ingeniero Gustavo Valera, el matemático Santiago Ortiz, el biólogo Ramón Guardans -quien creó la instalación interactiva Ecolocación algorítmica (2003) y donde colaboró MLM, Soundplots y el ZKM-, o el físico Abelardo Gil Fornier. Marcos García también participó como becario y mediador, junto a Laura Fernández, o Paqui Blanco, responsable de Intermediae. Fruto de algunas de las actividades también fueron los encuentros con los colectivos alternativos de la ciudad, coordinados por la historiadora y catedrática de la UNED, Yayo Aznar. Partiendo de unas relaciones conflictivas o de mutua indiferencia entre los colectivos y la institución, se consiguió generar un entorno de confianza y colaboración abierta, cuyo resultado fueron las Ayudas a la Creación Contemporánea del Ayuntamiento. Una fructífera convergencia de procesos Top down-Bottom up (Rico 2018). De ahí, se formó una de las derivadas de MLM, que fue el proyecto Intermediae-Matadero Madrid.

Muchos de los proyectos que fueron desarrollados en Medialab Madrid eran interdisciplinares y tuvieron tanta relevancia que fueron seleccionados en festivales y eventos de arte de los medios, como Ars Electrónica en Linz, International Symposium on Electronic Art (ISEA), o Ciberarts, entre otros.

Por ello, no solo se puede y se debe considerar su importancia en relación con las nuevas formas de aprendizaje y activismo, sino también desde el punto de vista de la misma ciudad de Madrid:

«[...] totalmente transformadora, ya que el centro no partía de un ecosistema cultural especialmente fuerte y cohesionado en la materia, más bien al contrario, y su aparición fue decisiva en la creación de ese ecosistema, cumpliendo una excelente función de dinamización, conexión y transformación de procesos y dinámicas». (Alsina 2018).

Actualmente que han desaparecido espacios como la Mediateca de CaixaForum, el Festival VIDA, el Festival ArtBots, la plataforma alepharts.org, la Gallery9 del WalkeArt Center (del máster en Digital Media de la UPF), el Centre for New Media Culture RIX, el Dutch Electronic Art Festival, la revista especializada A mínima (Iglesias 2018) y tantos otros espacios que están en vías de desaparición, artículos de similar naturaleza a éste son una de las iniciativas relevantes para que su memoria siga sobreviviendo.

\section{Referencias bibliográficas}

Fagerstrom, Asle; Arntzen, Erik y Gordon R. Foxall. 2015. «Use of MediaLab ${ }^{T M}$ in experimental behavioral research: The case of consumer decision making.» European Journal of Behavior Analysis, no. 102: 203-214. https://doi.org/10.1080/15021149 2009.11434319
Anderson, Chris. 2013. Makers: La Nueva Revolucion Industrial. Barcelona: Ediciones Urano.

Blessi, Giorgio Tavano, Sacco, Pier Luigi y Thomas Pilati. 2011. «Independent artist-run centres: an empirical analysis of the Montreal non-profit visual arts field». Cultural Trends. 20(2): 141-166. https://doi.org/10.1080/09548963.2011.563907

Brea, José Luis. 2002. La era postmedia. Acción comunicativa, prácticas (post)artísticas y dispositivos neomediales. Salamanca: Consorcio Salamanca.

Chatzichristodoulou, Maria. 2013. «New Media Art, Participation, Social Engagement and Public Funding». Visual Culture in Britain, 14(3): 301-318. https://doi.org/10.1080/14714787.2013.827486

Deitcher, David. 2000. «Tomar el control: arte y activismo». En Los manifiestos del arte posmoderno: textos de exposiciones, 19801995, coord. por Anna María Guasch Ferrer. Madrid: Akal.

Del Río, Victor. 2003. «El espacio ideológico del arte y la ciencia». Lápiz: Revista Internacional de Arte, no. 193: 28-39. http://www. victordelrio.net/PDFS/Ensayos/espacio\%20ideologico.pdf [Fecha de consulta: 16/05/2019]

Haste, H. 2009. "What is 'competence' and how should education incorporate new technology's tools to generate 'competent civic agents'». The Curriculum Journal, 20(3): 207-223. https://doi. org/10.1080/09585170903195845

Estalella, Adolfo; Rocha, Jara y Antonio Lafuente. 2013. «Laboratorios de procomún: experimentación, recursividad y activismo». Teknokultura 1(10): 21-48.

González, María. 2009. «New Educational Settings. Cognitive Challenges for the Realization of a Collective Intelligence». [Los nuevos entornos educativos: desafíos cognitivos para una inteligencia colectiva]. Comunicar, no. 33: 141-148. https://doi.org/10.3916/ c33-2009-03-005.

Laddaga, Reinaldo. 2011. Estética de laboratorio. Buenos Aires: Adriana Hidalgo editora.

Mullinax, M. 2014. «What we made: Conversations on art and social cooperation». Visual Studies 27(29): 112-113.

Ohlenschläger, Karin. 2007. Memoria de las actividades de MediaLab Madrid. Madrid: Centro Cultural Conde Duque, Ayuntamiento de Madrid, MedialabMadrid.

Ortega, José Felipe y Joaquín Rodríguez. 2011. El Potlatch digital. Wikipedia y el triunfo del procomún y el conocimiento compartido. Madrid: Cátedra.

Ortega, Inés y Reinaldo Villar. 2014. «El modelo Medialab: conceptos, contextos y clasificación. Posibilidades de una didáctica artística en el laboratorio revisado del laboratorio de medios». Revista Pulso, n. ${ }^{\circ}$ 37: 149-165.

Rodrigo, Javier. 2007. «De la intervención a la re-articulación: trabajo colaborativo desde políticas culturales». [Mensaje en un blog] http://javierrodrigomontero.blogspot.com/2010/04/de-laintervencion-la-re-articulacion.html. 


\title{
artnodes
}

http://artnodes.uoc.edu

MediaLab Madrid 2002-2006

Rodrigo, Javier. 2011. «Políticas de colaboración y prácticas culturales: redimensionar el trabajo del arte colaborativo y las pedagogías». [Mensaje en un blog] https://bit.ly/2sklrRs

Romero-Frías, Esteban, Robinson García, Nicolás. 2017. «Laboratorios sociales en universidades: Innovación e impacto en Medialab». Revista Comunicar, Vol. XXV, n. ${ }^{0} 51$.

Rosales, Carlos. 2009. «Aprendizaje formal e informal con medios Pixel-Bit». Revista de Medios y Educación, n. ${ }^{0}$ 35: 21-32. Universidad de Sevilla, España.

Salaris, Claudia. 1990. «Le futurisme et la publicité». En Art \& Pub. Art \& Publicité 1890-1990. París: Éditions du Centre Pompidou. Pp. 180-197.

Sánchez, José Manuel. 2011. La nueva ilustración: ciencia, tecnología y humanidades en un mundo interdisciplinar. Oviedo: Nobel.

Sangrà, Albert y Steve Wheeler. 2013. «Nuevas formas de aprendizaje informales: ¿0 estamos formalizando lo informal?» En: «La informalización de la educación». Revista de Universidad y Sociedad del Conocimiento (RUSC) 1(10): 107-115. http://dx.doi. org/10.7238/rusc.v10i1.1689

Serra, Artur. 2010. «Citilabs: ¿Qué pueden ser los laboratorios ciudadanos?» La Factoría: 45- 46.
Toffler, Alvin .1980. The Third Wave. Nueva York: William Morrow and Company, Inc.

V. AA. 2002. El número y la Mirada. Barbadillo y el Centro de Cálculo de la Universidad de Madrid. Córdoba: Caja San Fernando.

\section{Referencias entrevistas}

Alcalá, José Ramón. 2018. Entrevista realizada por las autoras con fecha 29/01/2018.

Alsina, Pau. 2018. Entrevista realizada por las autoras con fecha 09/02/2018.

Ohlenschläger, Karin. 2018. Entrevista realizada por las autoras con fecha 12/02/2018.

Ortiz, Santiago. 2018. Entrevista realizada por las autoras con fecha 21/02/2018.

Rico, Luis. 2018. Entrevista realizada por las autoras con fecha 25/02/2018.

\section{CV}

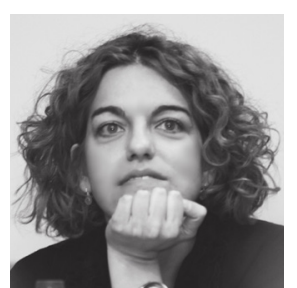

\author{
Raquel Caerols Mateo \\ Universidad Francisco de Vitoria \\ r.caerols.prof@ufv.es
}

Ctra. Pozuelo-Majadahonda KM 1.800

28223 Pozuelo de Alarcón (Madrid, España)

Acreditada como profesora titular de universidad por la Aneca y con un sexenio de investigación. Doctora en Creatividad Aplicada por la Facultad de Bellas Artes de la Universidad Complutense de Madrid (UCM). Profesora Adjunta en la Facultad de Comunicación de la Universidad Francisco de Vitoria en el área de creatividad. Además, ha participado como ponente en Congresos internacionales como el Computer Art en México DF, o el VI Congreso de lúdica y pedagogía. Por un nuevo aprender en Cartagena de Indias (Colombia). Cuenta con diversas publicaciones en prestigiosas publicaciones. Ha sido responsable de la dirección del I Simposio Cibercultura y New Media Art, financiado por el Ministerio de Cultura y Deporte del Gobierno de España. 


\section{artnodes}

http://artnodes.uoc.edu

MediaLab Madrid 2002-2006

\section{CV}

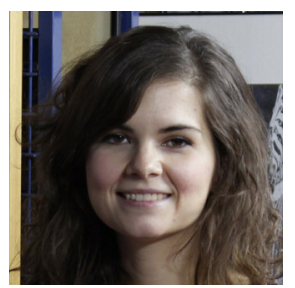

\section{Beatriz Escribano Belmar}

Universidad de Castilla la Mancha

beatriz.escribel@gmail.com

Calle Santa Teresa Jornet, s/n

16071 Cuenca

Beatriz Escribano Belmar, acreditada como Profesora Ayudante Doctora por la Aneca, obtuvo su doctorado en Arte y Nuevos Medios en 2017 con su tesis doctoral Copy Art Histories: The emergence of the photocopy machine in the 20th century art and its role as Historical Media Art. Tendencies and thematic cartography of Copy Art, sobre arte de los medios, electrografía artística y Copy Art. Licenciada en Bellas Artes (2011) por la Facultad de Bellas Artes de Cuenca, está especializada en el campo del arte y los nuevos medios desde el Máster de Investigación en Artes Visuales y Multimedia por la Universidad Politécnica de Valencia (2012). Tras ser beneficiaria de un contrato FPI por parte de la Junta de Comunidades de Castilla-La Mancha (2014-2017), ha sido investigadora postdoctoral y ha indagado la aportación española al arte de los medios y el rol de los procesos automáticos de [re] producción de imágenes surgido en los años 60 . Ha realizado diversas estancias en el Department for Image Science de la Danube University de Krems (Austria), en el Digital Media Lab de la Universität de Bremen (Alemania), en CITU Laboratoire Paragraphe de la Université Paris 8 de París (Francia) o en la Winchester School of Art (Gran Bretaña), entre otras, y ha sido coordinadora de la publicación Procesos: El Artista y la Máquina. Reflexiones en torno al Media Art histórico (2016). 